\title{
Nicho de mercado: El enfoque desde el océano azul
}

\author{
Carlos Chirinos \\ Universidad de Lima \\ Ingeniería Industrial n²9, 2011, ISSN 1025-9929, pp. 171-181 \\ Recibido: 4 de junio del 2011 / Aprobado: 19 de junio del 2011
}

\begin{abstract}
Resumen: En los últimos años el término "emprendedor" ha tomado mayor relevancia en nuestro hablar diario. Es habitual emplear este término para designar a una "persona que crea una empresa" o a quien empieza un proyecto por su propia iniciativa. Se ha sugerido que el ser emprendedor es una de las cualidades esenciales de un empresario u hombre de negocios, junto a la de innovación y organización. La primera etapa del emprendimiento es identificar el producto y el mercado al cual le venderemos un producto o servicio, tarea crucial para garantizar el éxito del proyecto. Dentro de este contexto aparece el concepto "nicho de mercado" como el espacio de mercado al que se dirigirá el proyecto de negocio.
\end{abstract}

Palabras clave: nichos de mercado / emprendedores / emprendimiento / estrategias en los negocios / estrategias de márketing / innovaciones

\section{The niche market, an approach from the blue ocean strategy}

AвsтRACт: Lately "Entrepreneurship" term has taken major relevancy in business environment. Entrepreneur is a person who is willing to launch a new venture or enterprise and accept full responsibility for the outcome. Entrepreneurship is one of the essential qualities of a businessman, the same as innovation and organization. The first stage of the Entrepreneurship is to identify the product and the market to which we will sell a product or service, this is the most important task to guarantee the success of the project. Inside this context there appears the concept "niche market" as the subset of the market on which a specific product is focusing.

Keywords: market niche / entrepreneurship / venture / business strategies / marketing strategies / innovations 


\section{NICHO DE MERCADO}

Un nicho de mercado es el término de mercadotecnia utilizado para referirse a la porción de un segmento de mercado en la que los individuos poseen características y necesidades semejantes, y donde estas últimas no están del todo cubiertas por la oferta general del mercado.

En este sentido, un nicho de mercado, debido a su pequeña dimensión, es generalmente despreciado por las grandes empresas, constituyendo, por ello, una excelente oportunidad para las pequeñas empresas, que allí pueden escapar del dominio de aquellas y lograr una posición de liderazgo.

\subsection{Características de un nicho de mercado}

En general, un nicho de mercado presenta las siguientes características:

- Es la fracción de un segmento de mercado. Es decir, cada nicho de mercado tiene algunas características similares a las del segmento de donde proviene pero a la vez presenta algunas particularidades que la distinguen de otros nichos o del segmento en su conjunto. Por ejemplo: las personas que frecuentan las discotecas para divertirse con los amigos los fines de semana representan un segmento de mercado. Por su parte, quienes además de ir a las discotecas eligen la zona VIP representan un nicho de mercado.

- Es un grupo reducido; lo componen personas, empresas u organizaciones en una cantidad pequeña (comparándola con el segmento de mercado al que pertenece). Por ejemplo, el número de jóvenes que eligen la zona VIP en una discoteca es mucho más reducido que la cantidad de personas que se divierten en la zona general.

- Tienen necesidades específicas y parecidas; por lo general, consisten en deseos muy particulares y que además tienen su grado de complejidad, por lo cual este grupo está dispuesto a pagar un "extra" para adquirir el producto o servicio que cumpla con sus expectativas. Por ejemplo, las personas que eligen la zona VIP en las discotecas tienen requerimientos muy especiales, como más espacio para bailar, mesas y asientos más cómodos o acolchados, un menú de comidas y 
bebidas más selecto, personal más calificado en atenderlos, música, video, zona especial para apreciar el salón de baile, etcétera.

- Existe la voluntad para satisfacer sus necesidades; es decir, presentan una buena predisposición por adquirir un producto o servicio que satisfaga sus expectativas, y en el caso de las empresas u organizaciones, tienen la capacidad de tomar decisiones de compra. Por ejemplo, las personas que se divierten en la zona VIP solicitan su entrada para esa categoría incluso sin necesidad de que el personal de la discoteca se lo sugiera.

- Existe la capacidad económica. Cada componente del nicho de mercado tiene la suficiente capacidad económica que le permite incurrir en los gastos necesarios para obtener el satisfactor de su necesidad o deseo. Incluso, están dispuestos a pagar un monto adicional al promedio con el fin de lograr una mejor satisfacción. Por ejemplo, las personas que elijen la zona VIP en cualquier discoteca tienen la suficiente fluidez económica como para pagar un monto extra por darse ese lujo.

- Requiere operaciones especializadas. Al existir necesidades o deseos con características específicas e incluso complejas, el nicho de mercado requiere de proveedores especializados y capaces de cubrir sus expectativas. Por ejemplo, los clientes de la zona VIP esperan que las discotecas les proporcionen una ventanilla exclusiva donde no tengan que hacer fila, un espacio más amplio y moderno, personal calificado para atenderlos, membrecías especiales, tarjetas de cliente VIP, etcétera.

- Existen muy pocas o ninguna empresa ofertante. En aquellos nichos de mercado donde las particularidades y la complejidad de sus necesidades o deseos requieren de una alta especialización, es normal que no existan más de una o dos empresas proveedoras, incluso puede darse el caso de que no exista ninguna.

- Tiene tamaño suficiente como para generar utilidades. Según Philip Kotler, un nicho de mercado ideal es aquel que tiene el tamaño necesario para ser rentable. 
Gráfico 1

Características de un nicho de mercado

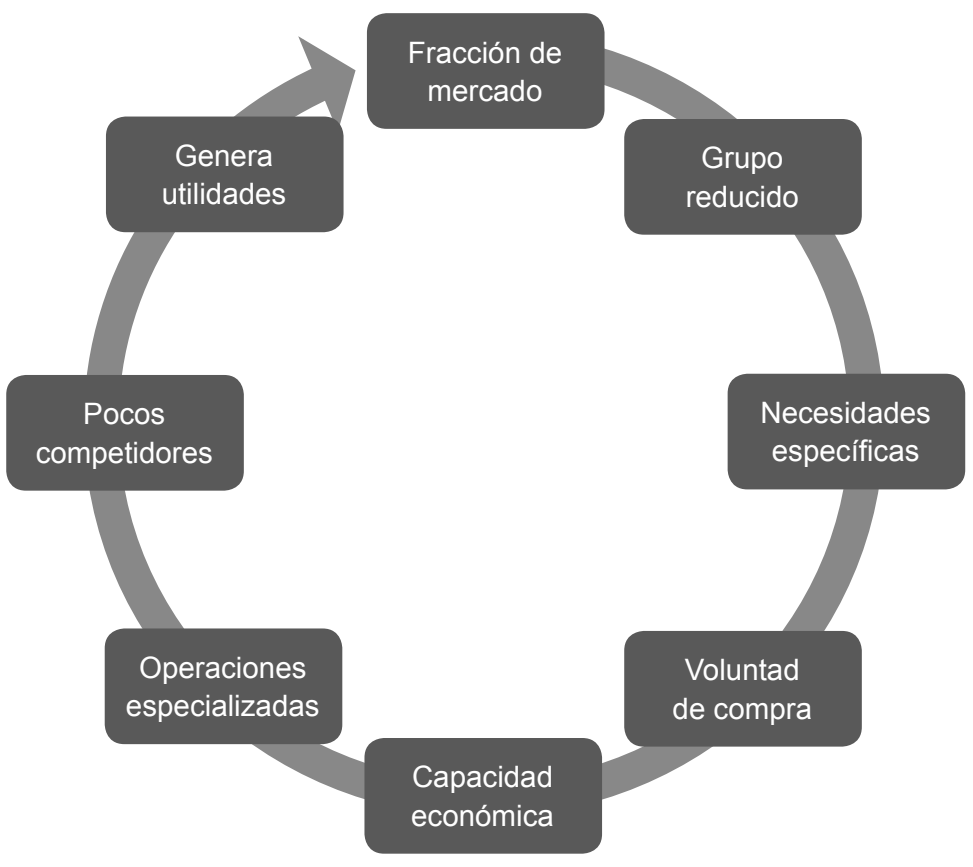

Elaboración propia.

\section{2 ¿Cómo identificar nuestro nicho de mercado?}

Hay muchos emprendedores que no consiguen fácilmente identificar su nicho de mercado en forma correcta. Ellos eligen un mercado que es inapropiado respecto a sus habilidades y recursos, o eligen un mercado que es demasiado amplio, por lo cual sus oportunidades de éxito son limitadas.

Existen diversas teorías para definir estrategias, segmentar mercados y buscar nichos, en este artículo proponemos la estrategia del océano azul como metodología para identificar un nicho dentro del mercado. Esta estrategia ha sido formulada por W. Chan Kim y Reneé Mauborgne en su libro titulado precisamente La estrategia del océano azul (2008). 


\section{La estrategia del océano azul}

Los mencionados autores afirman que a diferencia de lo que comúnmente se supone, la mejor estrategia para sacar del juego a la competencia no es competir directamente con esta, sino, por el contrario, no competir. En vez de tratar de superar a la competencia con el fin de obtener una porción de un mercado existente, "océano rojo", lo mejor es buscar un "océano azul", es decir, un mercado inexplorado que nadie haya tocado y que tenga el potencial de crecer.

La estrategia del océano azul lleva a las empresas a generar un nuevo espacio de mercado haciendo irrelevante la competencia, creando y capturando nueva demanda, alineando todas las actividades de la organización con el objetivo de procurar la disminución de costos y a la vez el aumento del valor de los productos.

Los océanos azules se caracterizan por la creación de mercados en áreas que no están explotadas en la actualidad, y que generan oportunidades de crecimiento rentable y sostenido a largo plazo. Hay océanos azules que no tienen nada que ver con las industrias actuales, aunque la mayoría surge de los océanos rojos al expandirse los límites de los negocios ya existentes. El hecho fundamental es que cuando aparecen los océanos azules, la competencia se torna irrelevante, pues ella está en otro mar y en este nuevo mar las reglas del juego están esperando a ser fijadas.

Visto de manera sintetizada, lo que la estrategia del océano azul plantea es buscar un producto o servicio con valor innovado, creando así un nuevo mar, es decir, un nuevo nicho. Por lo tanto, la tarea es doble: definir nuestro producto o servicio y determinar nuestro nicho de mercado.

\section{LA INNOVACIÓN DEL PRODUCTO. PASOS QUE SE DEBEN SEGUIR: ELIMINAR-REDUCIR, AUMENTAR, CREAR}

Chan y Mauborgne (2008) proponen cuatro preguntas orientadas a redefinir el producto o servicio ofrecido. Estas son:

- ¿Qué características de nuestros productos o servicios de aquellos que el mercado concibe como establecidos deben ser eliminadas?

- ¿Qué características deben ser reducidas a estándares menores a las aceptadas por el mercado? 
- ¿Qué características deben ser incrementadas por encima de los niveles considerados por el mercado?

- ¿Qué características nunca antes consideradas por el mercado deben ser creadas?

Este esquema permite construir una nueva curva de valor para el producto o servicio, considerando los elementos de valor del comprador.

\section{Gráfico 2}

Innovación del valor de un producto o servicio

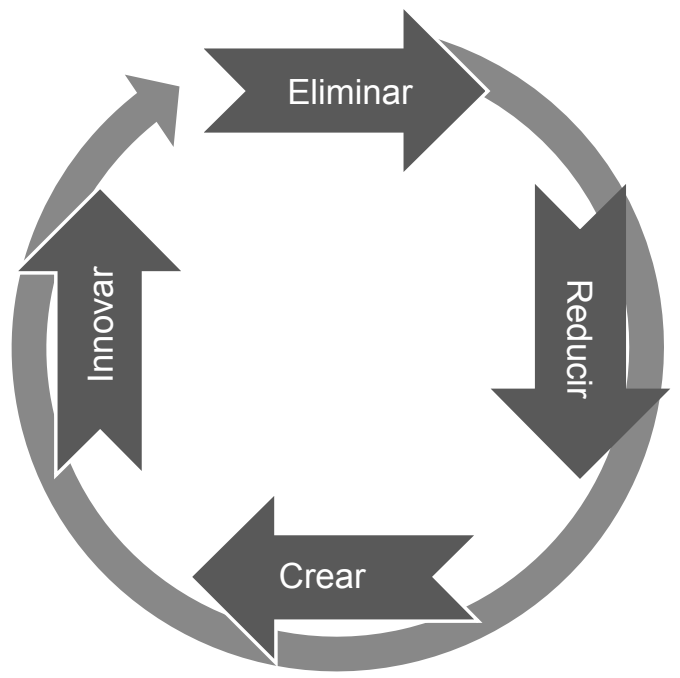

Elaboración propia.

\section{LOS NUEVOS CLIENTES}

Por otro lado, y para complementar la estrategia del océano azul, Chan y Mauborgne (2008) establecen que generalmente existen tres grupos de no-consumidores a los que una empresa puede intentar llegar:

- Los “casi clientes”, que están en el borde del segmento del mercado, casi a punto de entrar en el nicho.

- Los "que no desean ser clientes", que conscientemente eligen no formar parte del segmento de mercado.

- Los "no explorados", que no fueron considerados para este segmento de mercado en particular. 


\section{Gráfico 3}

Grupos de no consumidores

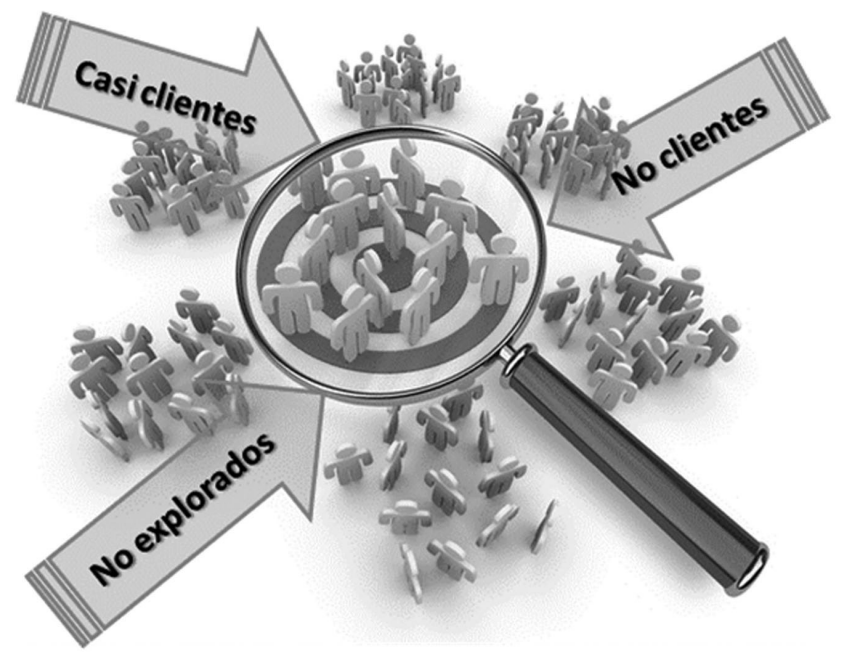

Elaboración propia.

Finalmente, es importante considerar que la estrategia del océano azul es más eficaz en dos tipos de mercados:

- Mercados donde el modelo de negocio esté en crisis. Por ejemplo, la música, el alquiler de videos o las cabinas de internet.

- Mercados altamente commoditizados. Es decir, donde todas las empresas ofrecen productos y servicios similares. Por ejemplo, el mercado de la telefonía móvil y las tarjetas de crédito.

\section{EL NICHO DE NINTENDO}

A continuación veamos el caso de Nintendo y la estrategia del océano azul.

Nintendo era solo un pequeño jugador de este terreno de competencia, luchando únicamente por mantenerse dentro del juego y estar al nivel de su competencia, hasta que un día decidió modificar su estrategia.

Durante mucho años, dos competidores lideraron el mercado de los videojuegos: Sony y Microsoft. Era una industria que parecía estar ce- 
rrada en lo que Chan y Mauborgne (2008) han descrito como el océano rojo de la competencia, donde el foco es una guerra en la que el único objetivo es ganar la próxima batalla, aumentar la participación en el mercado, atraer nuevos consumidores y obtener un mayor volumen de ventas que los competidores.

Así, todo era tecnología, sofisticados diseños de pantalla, gráficos modernos y alta resolución. Hasta el año 2006, Nintendo seguía un plan que no parecía darle buenos resultados. Playstation 2, de Sony, y Xbox, de Microsoft, estaban a la cabeza de la batalla tecnológica, mientras Nintendo trataba de sobrevivir con productos como GameCube, que poco tenían que ver con lo que se estaba viendo en el mercado. Parecía que tecnológicamente estaban muy atrasados como para salir a sorprender.

Sin embargo, Nintendo sí sorprendió, cambiando su estrategia y su foco de mercado para generar su propio océano azul. En la estrategia del océano azul no se compite por el mercado preexistente sino que se crea demanda con un producto innovado en un nuevo mercado, agrandando el mercado existente. El lanzamiento de la consola Wii fue exactamente eso: intentaron abrir sus esfuerzos para acceder a un mercado más amplio, centrándose en beneficios y atributos que no fueran solo tecnológicos.

Lo que llama la atención de esta nueva consola Wii es justamente que va más allá de la definición de un target de edad limitado. El foco que antes se reducía a sorprender a jóvenes adultos se amplió, y ahora incluye mujeres, niños, ancianos, y hasta como actividad para toda la familia; noción que hasta entonces había sido ignorada por Sony y Microsoft.

La consola Wii parece haber alcanzado por lo menos a los "casi clientes", pero sobre todo a los "no explorados", mientras que no perdió fuerzas tratando de llegar a los "no clientes".

Esta nueva estrategia de Nintendo significó un cambio en la carrera tecnológica que viró hacia el desarrollo de herramientas que hagan a los juegos más "amigables" y que terminen siendo más fáciles y divertidos para el usuario. Los controles inalámbricos abrieron infinitas opciones, y los accesorios para los controles permitieron una experiencia mucho más real de los juegos. 
Con una visión integrada de lo que significa una consola, Nintendo dio un giro a la industria y se hizo acreedora de un nuevo sector de la industria que mueve 30 mil millones de dólares.

Nintendo parece haber recuperado su estrellato y su fama como la número uno en videojuegos. La facilidad y realidad de los juegos se convierte en un atractivo para los usuarios, que, sumado al precio competitivo, llevó a que Nintendo esté vendiendo casi el doble que sus competidores conjuntamente.

$\mathrm{Al}$ crear un océano azul se puede descubrir un nuevo sector hasta entonces inexistente, o ampliar los límites de un océano rojo, creando un producto totalmente novedoso. En el primer caso, podemos considerar el ejemplo de eBay, que creó el mercado de las subastas on-line, y para el segundo, por ejemplo el caso de la consola Wii de Nintendo, que dentro del competitivo mundo de las consolas decidió posicionarse en la jugabilidad para cualquier tipo de público, en lugar de continuar la batalla por la mayor capacidad de proceso y los mejores gráficos.

\section{BIBLIOGRAFÍA}

Chan Kim, W. y Renèe Mauborgne (2008). La estrategia del océano azul. Bogotá: Norma.

Kotler, Philip (1996). Dirección de mercadotecnia. 8. ${ }^{a}$ edición. México D. F.: Prentice-Hall/Hispanoamericana.

Perú 21 (8 de mayo del 2010). "Busca nicho de mercado: especialízate". Sección Economía, p. 10. Lima.

Stanton, Etzel y Walker (2004). Fundamentos de marketing. 13. ${ }^{a}$ edición. México D. F.: McGraw-Hill.

Wikipedia. "Blue ocean strategy". <http://en.wikipedia.org/wiki/Blue_ Ocean_Strategy>. [Consulta: 30 de mayo del 2011.]

Wikipedia. "Emprendedor". <http://es.wikipedia.org/wiki/Emprendedor>. [consulta: 1 de mayo del 2011.]

Wikipedia. "Nicho de mercado". <http://es.wikipedia.org/wiki/Nicho_ de_mercado>. [Consulta: 1 de mayo del 2011.] 